$10-2011$

\title{
A Multi-Regulator Sliding Mode Control Strategy for Output- Constrained Systems
}

Hanz Richter

Cleveland State University, h.richter@csuohio.edu

Follow this and additional works at: https://engagedscholarship.csuohio.edu/enme_facpub

Part of the Aerospace Engineering Commons, and the Mechanical Engineering Commons How does access to this work benefit you? Let us know!

\section{Publisher's Statement}

NOTICE: this is the author's version of a work that was accepted for publication in Automatica. Changes resulting from the publishing process, such as peer review, editing, corrections, structural formatting, and other quality control mechanisms may not be reflected in this document. Changes may have been made to this work since it was submitted for publication. A definitive version was subsequently published in Automatica, 47,10, October 201, DOI: 10.1016/ j.automatica.2011.08.003

\section{Original Citation}

Richter, H., 2011, "A Multi-Regulator Sliding Mode Control Strategy for Output-Constrained Systems," Automatica, 47(10) pp. 2251-2259.

This Article is brought to you for free and open access by the Mechanical Engineering Department at EngagedScholarship@CSU. It has been accepted for inclusion in Mechanical Engineering Faculty Publications by an authorized administrator of EngagedScholarship@CSU. For more information, please contact library.es@csuohio.edu. 


\section{A multi-regulator sliding mode control strategy for output-constrained systems ${ }^{\text {kn }}$}

\author{
Hanz Richter* \\ Department of Mechanical Engineering, Cleveland State University, Cleveland, $\mathrm{OH}, \mathrm{USA}$
}

\section{Introduction}

This paper is concerned with the problem of control under output inequality constraints, relevant to practical situations where safety and other considerations require certain variables to be kept within allowable limits. The case where only one control input is available to handle both primary control and limit protection tasks is considered. This situation arises, for instance, in aircraft engine control, where thrust is the primary regulated variable, while outputs such as turbine temperature and compressor stall margin must be kept within limits (Jaw, 2009; Spang \& Brown, 1999). However, only fuel flow rate is available as a control input. In the absence of disturbance and with perfect model knowledge, limit protection can be easily guaranteed for the steady-state regime. For this, an allowable range for the setpoint of the regulated output is determined so that the corresponding steady values of the limited outputs remain within the desired limits. Limit violation, thus, will ensue at steady state if the regulated variable is driven to a setpoint outside the permissible range. There are two other circumstances that cause limits to be exceeded, namely transient violations and off-nominality. Indeed, limit preservation at steady-state does not imply that limited outputs remain within limits during their transition to

\footnotetext{
The material in this paper was partially presented at the 11th International Workshop on Variable Structure Systems, June 26-28, 2010, Mexico City, Mexico. This paper was recommended for publication in revised form by Associate Editor Henri Huijberts under the direction of Editor Andrew R. Teel.

* Tel.: +1 216687 5232; fax: +1216 5792474 .

E-mail address: h.richter@csuohio.edu.
}

the steady-state. Preclusion of such transient violations is not easily included as an objective in the controller design process. Off-nominality may arise from a change in controller tuning to address new performance requirements for the regulated variable, or from unintentional factors such as plant parameter variations or external disturbances.

The requirement that outputs of the form $y_{i}=C_{i} x$ remain within constant bounds at all times fits within the framework of control with state variable constraints using set invariance methods, a topic for which a vast amount of literature can be found. For an excellent survey, see Blanchini (1999). Under this paradigm, $x$ is required to belong to a set $H=\cap H_{i}$ at all times, where $H_{i}=\left\{x \in \mathbb{R}^{n}: C_{i}^{\prime} x \leq 1\right\}$ and $C_{i}^{\prime}$ are scaled versions of $C_{i}$. Many techniques require $H$ to be a convex set, which is not possible, for instance, when there are less limited output than states. Also, set invariance-based methods typically suffer from a tradeoff between conservativeness (i.e., designed controllers do not fully exploit the available limits) and computational complexity (i.e., a large number of decision inequalities must be evaluated in real-time). In the case of stateconstrained systems controlled with sliding modes, invariant set techniques have been developed by the author in Richter (2010) and Richter, O'Dell, and Misawa (2007). Constraint enforcement via model-predictive control, although effective, is widely-regarded as a computationally-intensive process, although efforts have been made to reduce its complexity (Bemporad, Borrelli, \& Morari, 2002a; Bemporad et al., 2002b; Richter, Singaraju, \& Litt, 2008). A number of "limit protection logic" schemes have been used for decades in the aerospace industry, but thorough analyses characterizing their behavior are not found in the open literature. One arrangement for a single output which must be precisely 


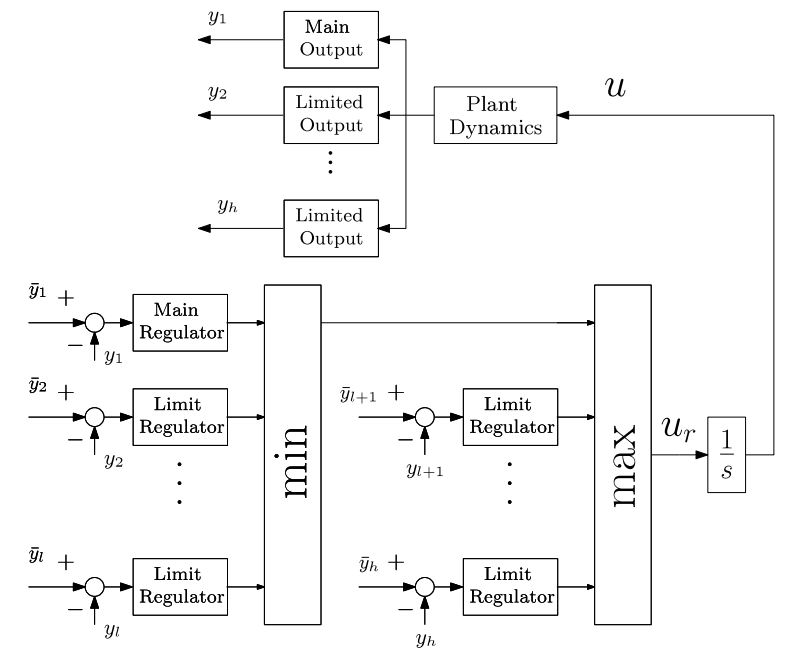

Fig. 1. Max-min multi-regulator system with integral control.

regulated from a single input, while keeping other outputs within limits is the multi-regulator scheme with integral control and min-max selectors (Jaw, 2009; Spang \& Brown, 1999) shown in Fig. 1. The regulators, thus, provide control input rates. Let $L=$ $\{1,2, \ldots, l\}$ and $H=\{l+1, l+2, \ldots, h\}$. The control rate applied to the integrator is selected as the maximum over the rates produced by the regulators in $L$ and the minimum over those produced by regulators in $H$. Thus, the max-min selection law is expressed as

$u_{r}=\max _{k \in H}\left\{\min _{j \in L}\left\{u_{r j}\right\}, u_{r k}\right\}$

where $u_{r j}$ are the min-selected regulator outputs and $u_{r k}$ are the max-selected regulator outputs. Throughout this paper, the subscript $r$ stands for 'rate', and should not be interpreted as an index. Some studies characterizing the behavior of schemes similar to the max-min arrangement have appeared, for instance in Åstrom and Hägglund (1995), Branicky (1994), Foss (1981) and Glattfelder and Schaufelberger (2003). More recently, the (nonasymptotic) stability of a similar scheme with linear regulators has been analyzed by Johansson (2003) using piecewise-quadratic Lyapunov functions. Even for linear regulators, a complete characterization of closed-loop behavior does not exist which includes essential issues such as determining which regulator will be active at the initial time and at steady-state, how to assign regulators to the max and min selectors, whether limit cycles exist, or how to design the regulators to address performance requirements. In the aircraft engine control field, where limit protection is indispensable, few works addressing the max-min arrangement have appeared (Litt et al., 2009; Spang \& Brown, 1999). Of particular importance is the observation that linear limit regulators may become active even when the auxiliary outputs are far from their limits, causing a degradation in the response of the main output due to an overriding control objective (Litt et al., 2009). This motivates the replacement of linear regulators by sliding mode controllers (SMC). In addition to the inherent robustness of SMC, this paper shows that using the differences between outputs and their allowable limits as sliding functions enables responses where the limits are maximally exploited, without excessive performance degradation in the response of the regulated variable.

\section{Problem statement and assumptions}

We consider linear single-input plants with integrated input given by the state-space description

$\dot{x}=A x+B u$ $\dot{u}=u_{r}$

where $x \in \mathbb{R}^{n}$, and $u$ and $u_{r}$ are scalars. Assume that a set of outputs is defined as

$y_{i}=G_{i} x+\Theta_{i} u$

for $i=1,2, \ldots, h$, with with $G_{i}$ an 1-by- $n$ vector and $\Theta_{i}$ an scalar. We make the following assumptions:

Assumption 1. $A$ is non-singular.

\section{Assumption 2. Define}

$A_{e q, i}=A-\frac{B G_{i}}{\Theta_{i}}$.

It is assumed that $\Theta_{i} \neq 0$ and matrices $A_{\text {eq }, i}$ have eigenvalues with negative real parts, for $i=1,2, \ldots, h$, that is, $y_{i}$ are minimumphase outputs of Eq. (2).

Note that when Assumption 1 fails due to a single zero eigenvalue, a straightforward modification of the results of this paper extends its applicability by elimination of input integration. The case $\Theta_{i}=$ 0 is discussed later.

\subsection{Control objectives}

Without loss of generality, let $y_{1}$ be the output whose setpoint is to be transferred with zero steady-state error. This must be achieved under constraints of the form $y_{k} \leq \bar{y}_{k}$ and $y_{l} \geq \bar{y}_{l}$, where $k$ are the indices of the upper-limited outputs and $l$ are the indices of the lower-limited outputs. In addition, usual transient response specifications may apply for the design of the main output regulator.

\subsection{Sliding mode control laws}

Although the multi-regulator arrangement with max-min selectors with linear regulators has been used in the aerospace field (Litt et al., 2009; Spang \& Brown, 1999), this paper replaces them with SMC regulators that introduce sliding modes at the limit boundaries to guarantee invariance and no conservativeness in exploiting the available limits. Define sliding variables as

$s_{i}=y_{i}-\bar{y}_{i}$

for $i \in L \cup H$, where $\bar{y}_{i}=G_{i} \bar{x}_{i}+\Theta_{i} \bar{u}_{i}$. The reference variables $\bar{x}_{i}$ and $\bar{u}_{i}$ are selected to be equilibrium pairs, that is, so that $A \bar{x}_{i}+B \bar{u}_{i}=0$. The standard SMC control law is obtained by requiring that $s_{i}=0$ in finite time (reaching phase). Beyond the reaching phase, $s_{i}=0$ must become invariant (sliding phase). The system then evolves with reduced-order dynamics matching the zero dynamics associated with output $s_{i}$. Thus, a minimum-phase assumption is required. The standard SMC literature (Edwards \& Spurgeon, 1998; Utkin, 1992) elaborates on the benefits associated with sliding modes, in particular, their trademark insensitivity to a class of disturbances and parametric uncertainties. For a single SMC regulator (fixed $i$ ), the control law given below in Eq. (7), where $\eta_{i}$ is a positive constant, forces the Lyapunov function $\frac{1}{2} s_{i}^{2}$ to have derivative $s_{i} \dot{s}_{i}=-\eta_{i} \operatorname{sign}\left(s_{i}\right)$, implying that the set $s_{i}=0$ is reached in finite-time, with subsequent invariance.

$u_{r i}=-\frac{1}{\Theta_{i}}\left(G_{i}(A x+B u)+\eta_{i} \operatorname{sign}\left(s_{i}\right)\right)$.

In view of the definition of $s_{i}$, a limit regulator, if operated alone, causes its corresponding limited output to attain the limit value in finite time without overshoot. Under the max-min selection logic, the closed-loop system is given by Eqs. (2)-(4), (6), (7) and (1). The controller implements Eqs. (6), (7), (1) and (3). 


\section{Behavior under a fixed regulator}

Let $i$ and $j$ be two fixed regulator indices and define the augmented state as $x_{a} \triangleq\left[x^{T} \mid u\right]^{T}$, let $\bar{x}_{a i}=\left[\bar{x}_{i}^{T} \mid \bar{u}_{i}\right]^{T}$ and define the augmented state relative to $i$ as $\tilde{x}_{a} \triangleq x_{a}-\bar{x}_{a i}$. Using this definition, it is straightforward to derive the following identities pertaining to system behavior under control law (7):

$$
\begin{aligned}
& \dot{\tilde{x}}_{a}=A_{i} \tilde{x}_{a}-\frac{1}{\Theta_{i}} B_{i} \eta_{i} \operatorname{sign}\left(s_{i}\right) \\
& s_{j}=J_{j} \tilde{x}_{a}+\Delta_{j, i} \\
& \dot{s}_{j \mid i}=\Theta_{j}\left(\Gamma_{j, i} \tilde{x}_{a}-\frac{\eta_{i}}{\Theta_{i}} \operatorname{sign}\left(s_{i}\right)\right)
\end{aligned}
$$

where:

$$
\begin{aligned}
& A_{i}=\left[\begin{array}{cc}
A & B \\
-\frac{G_{i}}{\Theta_{i}} A & -\frac{G_{i}}{\Theta_{i}} B
\end{array}\right], \quad B_{i}^{T}=\left[0_{1 \times n} \mid 1\right] \\
& J_{j}=\left[G_{j} \mid \Theta_{j}\right], \quad \Delta_{j, i}=J_{j}\left(\bar{x}_{a i}-\bar{x}_{a j}\right) \\
& \Gamma_{j, i}=\left[\frac{G_{j}}{\Theta_{j}}-\frac{G_{i}}{\Theta_{i}}\right][A \mid B] .
\end{aligned}
$$

The notation $\dot{s}_{j \mid i}$ is interpreted as "the derivative of $s_{j}$ when $i$ is the active regulator". When $i=j$ we simply write $\dot{s}_{i}$. Note that $\Delta_{i, i}=0$ and $\Gamma_{i, i}=0$ for $i \in L \cup H$. It is a standard fact of sliding mode theory that for each $i$, the spectrum of $A_{i}$ is formed by the eigenvalues of $A_{e q, i}$ from Eq. (5) and zero. The closed-loop system resulting from applying input (7) to system (2), (3) is more conveniently described in terms of the derivatives of the $s$ variables, as before, and the rate of $x$. In fact, define $X_{r} \triangleq \dot{x}$. The closed-loop system dynamics are expressed as

$$
\begin{aligned}
& \dot{X}_{r}=A_{e q, i} X_{r}-B \frac{\eta_{i}}{\Theta_{i}} \operatorname{sign}\left(s_{i}\right) \\
& \dot{s}_{j \mid i}=\Theta_{j}\left[\left(\frac{G_{j}}{\Theta_{j}}-\frac{G_{i}}{\Theta_{i}}\right) X_{r}-\frac{\eta_{i}}{\Theta_{i}} \operatorname{sign}\left(s_{i}\right)\right] .
\end{aligned}
$$

The rate system is a convenient description, due to $A_{e q, i}$ being Hurwitz. It allows us to describe asymptotic properties. However, $s_{i}$ cannot be written as a function of $X_{r}$ in a manner analogous to Eq. (9). For this reason, both descriptions of the closed-loop dynamics will be used for different purposes, as convenient.

\subsection{Characterization of the equilibrium point}

Define a switching function $q(x, u)$ with values in $L \cup H$. The minimum (min), maximum ( $\max$ ) switching functions are expressed by Eqs. (16) and (17), respectively.

$$
\begin{aligned}
& q_{\min }=\underset{i \in L}{\arg \min }\left\{u_{r i}\right\} \\
& q_{\max }=\underset{j \in H}{\arg \max }\left\{u_{r j}\right\} .
\end{aligned}
$$

When the above equations yield non-unique values, an assignment is made according to a pre-defined arbitrary rule. For the remainder of this paper, $q_{\min }=\min (i, j)$ and $q_{\max }=\min (i, j)$ are assumed whenever $u_{r i}=u_{r j}$. When a max-min arrangement is used, it is assumed that the min preselection is applied to the first port of the max selector, so that the min input is used in case of equality with the max preselection. These assumptions will be referred to as default index assumptions.

Proposition 3. Under the min switching law, system (2), (3) has a unique equilibrium point at $\left(\bar{x}_{i^{*}}, \bar{u}_{i^{*}}\right)$, where $i^{*} \in L$ is the index s.t. $\frac{\operatorname{sign}\left(\Delta_{j, i^{*}}\right)}{\Theta_{j}} \leq 0 \forall j \in L$.

Proof. The equilibrium point requirements $\dot{u}=0$ and $A x+B u=0$ imply that $u_{r q}=0$, where $q$ is the index of the active regulator. Also, from Eq. (7), it is clear that $s_{q}=0$ at equilibrium. Now, for $q$ to be the active regulator, it is necessary that $u_{r q} \leq u_{r j}$ for all $j \in L$. At equilibrium this implies that $q$ is such that $-\frac{\eta_{q}}{\Theta_{q}} \operatorname{sign}\left(s_{q}\right)=$ $0 \leq-\frac{\eta_{j}}{\Theta_{j}} \operatorname{sign}\left(\Delta_{j, q}\right)$ for all $j \in L$. Since this inequality reduces to the selection of the minimum of a set of numbers with a default selection applicable in the case of equality, index $q=i^{*}$ is uniquely defined and it always exists. Define now $\tilde{x}_{a}$ relative to $i^{*}$. It is clear that $\dot{\tilde{x}}_{a}=0$ at equilibrium. Using Eqs. (8) and (9) together with the facts that all $\left(x_{i}, u_{i}\right)$ are equilibrium pairs and that $A_{e q, i^{*}}$ is nonsingular, we have $\tilde{x}_{a}=0$, implying that $\left(\bar{x}_{i^{*}}, \bar{u}_{i^{*}}\right)$ is the equilibrium point. Thus, $0 \leq-\frac{\operatorname{sign}\left(\Delta_{j, i^{*}}\right)}{\Theta_{j}}$ for all $j \in L$.

Note that the existence of a time $t_{r} \geq 0$ such that $q(t)=i^{*}$ for all $t \geq t_{r}$ has not yet been established. Indeed, the fact that $q$ remains constant once $\dot{x}=0=\dot{u}$ is shown later.

Given system parameters, it is straightforward to compute the terminal regulator index. All $\Delta_{j, i}$ combinations are computed. For the min law, an index $i^{*}$ is sought that satisfies $0 \leq-\frac{\eta_{j}}{\Theta_{j}} \operatorname{sign}\left(\Delta_{j, i^{*}}\right)$ for all $j \in L, j \neq i^{*}$. The determination of the terminal index for the max and max-min switching laws are presented next.

Proposition 4. Under the max switching law, system (2), (3) has a unique equilibrium point at $\left(\bar{x}_{i^{*}}, \bar{u}_{i^{*}}\right)$, where $i^{*} \in H$ is the index s.t. $\frac{\operatorname{sign}\left(\Delta_{\left.j, i^{*}\right)}\right.}{\Theta_{j}} \geq 0 \forall j \in H$. This index is termed terminalregulatorindex.

The proof of this proposition is similar to that of Proposition 3.

Proposition 5. Under the max-min switching law of Eq. (1), system (2), (3) has a unique equilibrium point at $\left(\bar{x}_{i^{*}}, \bar{u}_{i^{*}}\right)$, where $i^{*} \in L \cup H$ is the index satisfying condition (18):

$0 \geq-\frac{\operatorname{sign}\left(\Delta_{k, i^{*}}\right)}{\Theta_{k}} \quad \forall k \in H$

and either condition (19) or condition (20):

$0 \leq-\frac{\operatorname{sign}\left(\Delta_{j, i^{*}}\right)}{\Theta_{j}} \quad \forall j \in L$

$0>\min _{j \in L}\left\{-\frac{\operatorname{sign}\left(\Delta_{j, i^{*}}\right)}{\Theta_{j}}\right\}$.

When condition (19) is satisfied, the terminal regulator index $i^{*} \in L$. Otherwise, condition (20) is satisfied and $i^{*} \in H$.

Proof. Let $i^{*}$ be the steady regulator. For $i^{*} \in L$, it is necessary and sufficient that $0=u_{r i *} \leq u_{j} \forall j \in L\left(i^{*}\right.$ wins within $\left.L\right)$ and $0=u_{r i} \geq u_{r h} \forall h \in H$ ( $L$ set wins). The first condition corresponds to inequality (19), while the second one to inequality (18). On the other hand, for $i^{*} \in H$, it is necessary and sufficient that $0=u_{r i^{*}} \geq$ $u_{h} \forall h \in H\left(i^{*}\right.$ wins within $\left.H\right)$ and $0=u_{r i^{*}}>\min _{l \in L}\left\{u_{r l}\right\}$. These conditions correspond to inequalities (20) and again, inequality (18).

A simple algorithm to identify the ending regulator $i^{*}$ in the max-min case can also be established.

\section{Stability proof}

Unlike single-regulator sliding mode control schemes, one may not use $s_{q}^{2}$ for some fixed $q$ as a Lyapunov function showing global attractiveness of the set $s_{q}=0$. Even for $q=i^{*}$, it is easy to find a simulation counter-example showing that $s_{q}^{2}$ is nonmonotonically decreasing towards zero. Moreover, the multiple Lyapunov approaches of Branicky (1998), and even the much less restrictive approach of Zhao and Hill (2008), are difficult to apply. In the first case, one must prove that $s_{j}^{2}$ is monotonically decreasing in intervals during which $q(t)=j$ and that the sequence $s^{2}\left(t_{j}\right)$ is 
decreasing, where $t_{j}$ is the sequence of times at which $j$ is switched on. In the second case, monotonicity is no longer required during the active intervals, and the $s^{2}\left(t_{j}\right)$ may be increasing, but must be bounded by a function satisfying certain requirements. In this paper, a proof of global asymptotic convergence to the equilibrium point $\bar{x}_{a i^{*}}$ is developed which relies only on Assumptions 1 and 2 . The proof is based on attractiveness properties of each individual sliding set, together with considerations about the geometry of the regions of $\mathbb{R}^{n+1}$ in which each regulator is active under any of the three switching laws.

\subsection{Stability: min switching}

Given a fixed $i \in L$, define the following sets

$$
\begin{aligned}
& \mathcal{R}_{i}^{+}=\left\{\tilde{x}_{a} \mid u_{r i} \leq u_{r j}, s_{i}>0, \forall j \in L\right\} \\
& \mathcal{R}_{i}^{-}=\left\{\tilde{x}_{a} \mid u_{r i} \leq u_{r j}, s_{i}<0, \forall j \in L\right\} \\
& \mathcal{R}_{i}^{0}=\left\{\tilde{x}_{a} \mid u_{r i} \leq u_{r j}, s_{i}=0, \forall j \in L\right\} \\
& \mathcal{R}_{j}=\mathbb{R}^{n+1} \backslash\left(\mathcal{R}_{i}^{+} \cup \mathcal{R}_{i}^{-} \cup \mathcal{R}_{i}^{0}\right)
\end{aligned}
$$

where \denotes set difference.

Proposition 6. The collection $\left\{\mathcal{R}_{i^{*}}^{+}, \mathcal{R}_{i^{*}}^{-}, \mathcal{R}_{i^{*}}^{0}, \mathcal{R}_{j}\right\}$ is a partition of $\mathbb{R}^{n+1}$ and $0 \in \mathcal{R}_{i^{*}}^{0}$.

Proposition 6 follows directly from the fact that $s_{i^{*}}=0$ defines a hyperplane dividing $\mathbb{R}^{n+1}$ into three disjoint regions.

\section{Lemma 7. The following statements hold:}

(1) The set $\mathcal{R}_{i^{*}}^{0}$ is invariant, that is, if $\exists t_{r} \geq 0$ s.t. $\tilde{x}_{a}\left(t_{r}\right) \in \mathcal{R}_{i^{*}}^{0}$, then $\tilde{x}_{a}(t) \in \mathcal{R}_{i^{*}}^{0}$ for $t \geq t_{r}$. In addition, $\tilde{x}_{a}(t) \rightarrow 0$ as $t \rightarrow \infty$.

(2) If $\tilde{x}_{a}\left(t_{1}\right) \in \mathcal{R}_{i^{*}}^{+} \cup \mathcal{R}_{i^{*}}^{-}$for some $t_{1} \geq 0$, then $\exists t_{2} \geq t_{1}$ s.t. $\tilde{x}_{a}\left(t_{2}\right) \in \mathcal{R}_{i^{*}}^{0}$.

(3) If $\tilde{x}_{a}\left(t_{1}\right) \in \mathcal{R}_{j}$ for some $t_{1} \geq 0$, then $\exists t_{2} \geq t_{1}$ s.t. $\tilde{x}_{a}\left(t_{2}\right) \in$ $\mathcal{R}_{i^{*}}^{+} \cup \mathcal{R}_{i^{*}}^{-} \cup \mathcal{R}_{i^{*}}^{0}$.

Proof. Proof of 1: Suppose $\tilde{x}_{a}\left(t_{r}\right) \in \mathcal{R}_{i^{*}}^{0}$ for some $t_{r} \geq 0$. Then $s_{i^{*}}\left(t_{r}\right)=J_{i^{*}} \tilde{x}_{a}\left(t_{r}\right)=0$ and $\dot{s}_{i^{*}}\left(t_{r}\right)=\eta_{i^{*}} \operatorname{sign}\left(s_{i^{*}}\left(t_{r}\right)\right)=0$. Also, $u_{r^{*}}\left(t_{r}\right) \leq u_{r j}\left(t_{r}\right)$. We need to verify that $u_{r i^{*}}(t) \leq u_{r j}(t)$ and $s_{i *}(t)=0$ for $t \geq t_{r}$. Two possibilities exist: either there is no mode change, i.e., $q(t)=i^{*}$ for $t \geq t_{r}$, or a mode change exists at some time $t_{1}>t_{r}$. Suppose, first, that $q(t)=i^{*}$ (and therefore $\left.s_{i^{*}}(t)=0\right)$ for $t_{r} \leq t<t_{1}$ and that $q\left(t_{1}\right)=j$ for some $j \neq i^{*}$. Since $s_{k}$ are continuous functions of time for all $k$, we must have that $s_{i^{*}}\left(t_{1}\right)=0$. The change from mode $i^{*}$ to mode $j$ at $t_{1}$ requires that $u_{r i^{*}}(t) \geq u_{r j}(t)$ for $t \rightarrow t_{1}^{+}$and $u_{r i^{*}}(t) \leq u_{r j}(t)$ for $t \rightarrow t_{1}^{-}$. With a slight abuse of notation, this means

$$
\begin{aligned}
& \Gamma_{j, i^{*}} \tilde{x}_{a}\left(t_{1}^{-}\right) \leq-\frac{\eta_{j}}{\Theta_{j}} \operatorname{sign}\left(s_{j}\left(t_{1}^{-}\right)\right) \\
& \Gamma_{j, i *} \tilde{x}_{a}\left(t_{1}^{+}\right) \geq-\frac{\eta_{j}}{\Theta_{j}} \operatorname{sign}\left(s_{j}\left(t_{1}^{+}\right)\right) .
\end{aligned}
$$

Since the left-hand sides of inequalities (25) and (26) are also continuous at $t_{1}$ and $\eta_{j}>0$, it is necessary that

$$
-\frac{1}{\Theta_{j}} \operatorname{sign}\left(s_{j}\left(t_{1}^{-}\right)\right) \geq-\frac{1}{\Theta_{j}} \operatorname{sign}\left(s_{j}\left(t_{1}^{+}\right)\right) \text {. }
$$

Although $s_{j}$ is continuous, it is the argument of a discontinuous function. It can be directly verified that strict inequality in (27) can only be satisfied if $s_{j}\left(t_{1}\right)=0$, which implies $\dot{s}_{i^{*} j}\left(t_{1}\right)=0$ from Eqs. (9) and (10). If equality is considered, we also have, using $\Gamma_{i^{*}, j} \tilde{x}_{a}=$ $-\Gamma_{j, i^{*}} \tilde{x}_{a}$ in Eq. (10), that $\dot{s}_{i^{*} j}\left(t_{1}\right)=0$. Thus, $s_{i}^{*}(t)=0$ for $t_{r} \leq t \leq t_{1}$ and it can be directly verified that the condition $u_{r^{*}}\left(t_{1}\right) \leq u_{r j}\left(t_{1}\right)$ holds, contradicting the assumption that $q\left(t_{1}\right)=j \neq i$. Therefore $q(t)=i^{*}$ and $s_{i^{*}}(t)=0$ for $t \geq t_{r}$, verifying invariance. Asymptotic convergence of $X_{r}$ to zero is immediate from Eq. (14). Since $s_{i^{*}}=$ $J_{i} * \tilde{x}_{a}=0$ for $t \geq t_{r}$ and $X_{r}=[A \mid B] \tilde{x}_{a}$, it is evident that $\tilde{x}_{a}(t) \rightarrow 0$ as $t \rightarrow \infty$.

Proof of 2: Suppose $\tilde{x}_{a}\left(t_{1}\right) \in \mathcal{R}_{i^{*}}^{+} \cup \mathcal{R}_{i^{*}}^{-}$for some $t_{1} \geq 0$. Since $\mathcal{R}_{i^{*}}^{+} \cap \mathcal{R}_{i^{*}}^{-}=\emptyset$, suppose first that $\tilde{x}_{a}\left(t_{1}\right) \in \mathcal{R}_{i^{*}}^{+}$. This implies $q\left(t_{1}\right)=i^{*}$. If there is no mode change, that is, $q(t)=i^{*}$ for $t \geq t_{1}$, we have $\dot{s}_{i^{*}}(t)=-\eta_{i^{*}} \operatorname{sign}\left(s_{i^{*}}(t)\right)$ so $\exists t_{2} \geq t_{1}$ s.t. $s_{i^{*}}\left(t_{2}\right)=0$, that is, $\tilde{x}_{a}\left(t_{2}\right) \in \mathcal{R}_{i^{*}}^{0}$. Suppose now, by contradiction, that $q\left(t^{\prime}\right)=j \neq i^{*}$ for some $t^{\prime} \geq t_{1}$. Following an argument analogous to the proof of the first statement of this lemma, it can be deduced that $s_{j}\left(t^{\prime}\right)=0$ and that $\dot{s}_{i^{*} \mid j}\left(t^{\prime+}\right)=0$. Noting that

$u_{r i^{*}}-u_{r j}=\Gamma_{j, i^{*}} \tilde{x}_{a}+\frac{\eta_{j}}{\Theta_{j}} \operatorname{sign}\left(s_{j}\right)-\frac{\eta_{i^{*}}}{\Theta_{i^{*}}} \operatorname{sign}\left(s_{i^{*}}\right)$.

Eq. (10) shows that $u_{r i^{*}}\left(t^{\prime+}\right)=u_{r j}\left(t^{\prime+}\right)$, contradicting the existence of a mode change from $i^{*}$ to $j$ at $t=t^{\prime}$. Therefore the only possibility is that $\tilde{x}_{a}\left(t_{2}\right) \in \mathcal{R}_{i^{*}}^{0}$ for some finite $t_{2} \geq t_{1}$.

Proof of 3: Proceeding by induction, we first show that the statement is true for $l=2$. Suppose $\tilde{x}_{a}\left(t_{1}\right) \in \mathcal{R}_{j}$ for some $t_{1} \geq 0$. Then $q\left(t_{1}\right)=j \neq i^{*}$. Suppose, by contradiction, that $q(t)=j$ for all $t \geq t_{1}$. Then the system would behave as if it were controlled by single sliding-mode regulator, reaching the set $s_{j}=0$ in finite time and entering a sliding mode defined by Eq. (14). It can be directly verified that $\bar{x}_{a j}$ would meet the definition of an equilibrium point. If $\bar{x}_{a j}=\bar{x}_{a i^{*}}$, then $\Delta_{j, i^{*}}=0$ and $s_{i}^{*}=0$, so $j$ and $i^{*}$ would both satisfy the min selection conditions, since $u_{r i *}=u_{r j}$. This contradicts the uniqueness of the terminal regulator index. If $\bar{x}_{a j} \neq \bar{x}_{a i^{*}}$, the uniqueness of the equilibrium point is contradicted. Therefore, $q$ must switch to $i^{*}$ at some finite $t_{2} \geq t_{1}$, implying $\tilde{x}_{a}\left(t_{2}\right) \in \mathcal{R}_{i^{*}}^{+} \cup \mathcal{R}_{i^{*}}^{-} \cup \mathcal{R}_{i^{*}}^{0}$. Now suppose that the statement holds for $l-1$ regulators. We wish to show that it must hold for $l$ regulators. Define $L_{l-1}=\{1,2, \ldots, l-1\}$. Let $k^{*}$ denote the terminal index in $L_{l-1}$ and $i^{*}$ denote the terminal index in $L_{l}$. Suppose $\tilde{x}_{a}\left(t_{1}\right) \in \mathcal{R}_{j}$ for some $t_{1} \geq 0$. Then $q\left(t_{1}\right)=j \neq i^{*}$ for some $j \in L_{l}$. Suppose, again by contradiction, that $q(t)=j$ for all $t \geq t_{1}$. Then the system would behave as if only $l-1$ regulators existed. By the inductive hypothesis, $q\left(t_{2}\right)=k^{*}$ for some finite $t_{2} \geq t_{1}$, implying $\tilde{x}_{a}\left(t_{2}\right) \in \mathcal{R}_{k^{*}}^{+} \cup \mathcal{R}_{k^{*}}^{-} \cup \mathcal{R}_{k^{*}}^{0}$. We have thus shown that, if switching is restricted to occur in a subset of indices, $q$ must eventually switch to the terminal index in that subset, concluding the proof.

Theorem 8. Under Assumptions 1 and 2, all trajectories of System (2), (3) under control input (7) and the min switching law converge asymptotically to the unique equilibrium point $\bar{x}_{a i *}$.

Proof. Suppose first that $\tilde{x}_{a}(0) \in \mathcal{R}_{i^{*}}^{0}$. Then the first statement of Lemma 7 proves the theorem. Now suppose that $\tilde{x}_{a}(0) \in \mathcal{R}_{i^{*}}^{+} \cup$ $\mathcal{R}_{i^{*}}^{-}$. The second and first statements of Lemma 7 are applied to prove asymptotic stability. The only remaining possibility is, by the partition property of Proposition 6 , that $\tilde{x}_{a}(0) \in \mathcal{R}_{j}$. The third statement of Lemma 7 establishes that $q=i^{*}$ in finite time. If $s_{i^{*}}=0$ at this time, the first statement proves asymptotic stability. If not, the second statement followed by the first proves the desired result.

\subsection{Stability: max switching}

Noting that $\max \left\{u_{r i}\right\}=-\min \left\{-u_{r i}\right\}$, it is evident that the stability proof holds under max switching, since the validity of the statements of Lemma 7 is independent of the direction of the inequalities used in the proofs. 


\subsection{Stability: $\max -\min$ switching}

The max-min case requires additional analysis, as index selection cannot be expressed in terms of min only. However, the property $\max \left\{a_{k}-b_{j}\right\}=\max \left\{a_{k}\right\}-\min \left\{b_{j}\right\}$ for any two collections of numbers $\left\{a_{k}\right\}$ and $\left\{b_{j}\right\}$ proves useful in reducing the proof to the already-studied min and max cases. An important property of the max-min arrangement is that there exists a finite time after which switching is restricted to happen either among the min or the max selectors, whichever group contains the terminal index. Indeed, Proposition 5 shows that there is a unique terminal regulator index corresponding to a unique equilibrium point. For the remainder of the paper, and without loss of generality, it is assumed that the terminal regulator index belongs to the min set, that is, $i^{*} \in L$.

Lemma 9. Suppose $i \in L$ and $j \in H$ are such that $q(t)=i$ for $t<t_{1}$ and $q(t)=j$ for $t \geq t_{1}$, for some $t_{1}>0$. That is, suppose a mode change exists at $t_{1}$ from a min-selected regulator to a max-selected regulator. Then $s_{j}\left(t_{1}\right)=0$.

Proof. Let $j^{\prime}=\arg \max _{h \in H}\left\{u_{r h}(t)\right\}$ for $t<t_{1}$ and $i^{\prime}=\arg$ $\min _{l \in L}\left\{u_{r l}(t)\right\}$ for $t \geq t_{1}$. That is, $j^{\prime}$ denotes the regulator preselected by max when $i$ is active, and $i^{\prime}$ denotes the regulator preselected by min when $j$ is active. Using the notations $t_{1}^{-}$and $t_{1}^{+}$for instants of time before and after the mode change, we must have $u_{r j^{\prime}}\left(t_{1}^{-}\right) \leq u_{r i}\left(t_{1}^{-}\right)$and $u_{r i}\left(t_{1}^{-}\right) \leq u_{r i^{\prime}}\left(t_{1}^{-}\right)$. Non-strict inequality is used due to default selection assumptions. Also, $u_{r j^{\prime}}\left(t_{1}^{-}\right) \geq u_{r j}\left(t_{1}^{-}\right)$, therefore one has $u_{r j}\left(t_{1}^{-}\right) \leq u_{r i^{\prime}}\left(t_{1}^{-}\right)$. After the postulated mode change one must have $u_{r j}\left(t_{1}^{+}\right)>u_{r i^{\prime}}\left(t_{1}^{+}\right)$, where strict inequality is required for the change to occur, overriding the default selection. In summary, the following inequalities are relevant

$u_{r j}\left(t_{1}^{-}\right) \leq u_{r i^{\prime}}\left(t_{1}^{-}\right)$

$u_{r j}\left(t_{1}^{+}\right)>u_{r i^{\prime}}\left(t_{1}^{+}\right)$.

Using the identity $u_{r j}-u_{r i}=\Gamma_{i, j} x_{a}+\frac{\eta_{i}}{\Theta_{i}} \operatorname{sign}\left(s_{i}\right)-\frac{\eta_{j}}{\Theta_{j}} \operatorname{sign}\left(s_{j}\right)$ and continuity of $x_{a}$ to write $\Gamma_{i^{\prime}, j} x_{a}\left(t_{1}^{-}\right)=\Gamma_{i^{\prime}, j} x_{a}\left(t_{1}^{+}\right)$, inequalities (28) and (29) are combined to yield

$$
\begin{aligned}
& \frac{\eta_{j}}{\Theta_{j}} \operatorname{sign}\left(s_{j}\left(t_{1}^{-}\right)\right)-\frac{\eta_{i^{\prime}}}{\Theta_{i^{\prime}}} \operatorname{sign}\left(s_{i^{\prime}}\left(t_{1}^{-}\right)\right) \\
& >\frac{\eta_{j}}{\Theta_{j}} \operatorname{sign}\left(s_{j}\left(t_{1}^{+}\right)\right)-\frac{\eta_{i^{\prime}}}{\Theta_{i^{\prime}}} \operatorname{sign}\left(s_{i^{\prime}}\left(t_{1}^{+}\right)\right) .
\end{aligned}
$$

Since the four variable terms in inequality (30) undergo only discrete changes corresponding to zero crossings of the $s$-variable, it is clear that $s_{i^{\prime}}\left(t_{1}^{-}\right)=s_{i^{\prime}}\left(t_{1}^{+}\right)=s_{i^{\prime}}\left(t_{1}\right)=0$ or $s_{j}\left(t_{1}^{-}\right)=s_{j}\left(t_{1}^{+}\right)=$ $s_{j}\left(t_{1}\right)=0$, where continuity of $s$ has also been used. Suppose, by contradiction, that $\operatorname{sign}\left(s_{j}\left(t_{1}^{-}\right)\right)=\operatorname{sign}\left(s_{j}\left(t_{1}^{+}\right)\right)$. Then, it must be that $s_{i^{\prime}}\left(t_{1}^{-}\right)=s_{i^{\prime}}\left(t_{1}^{+}\right)=s_{i^{\prime}}\left(t_{1}\right)=0$. Since $i$ is active at $t_{1}^{-}$, $\dot{s}_{i}\left(t_{1}^{-}\right)=-\eta_{i} \operatorname{sign}\left(s_{i}\left(t_{1}^{-}\right)\right)=0$. It now follows that

$\dot{s}_{i^{\prime} \mid j}\left(t_{1}^{+}\right) / \Theta_{i}^{\prime}=\Gamma_{i^{\prime}, j} x_{a}\left(t_{1}^{+}\right)-\eta_{j} \operatorname{sign}\left(s_{j}\left(t_{1}^{+}\right)\right) / \Theta_{j}$

$\dot{s}_{i^{\prime} \mid j}\left(t_{1}^{+}\right) / \Theta_{i}^{\prime}=\Gamma_{i^{\prime}, j} x_{a}\left(t_{1}^{-}\right)-\eta_{j} \operatorname{sign}\left(s_{j}\left(t_{1}^{-}\right)\right) / \Theta_{j}$

$\dot{s}_{i^{\prime} \mid j}\left(t_{1}^{+}\right) / \Theta_{i}^{\prime}=u_{r j}\left(t_{1}^{-}\right)-u_{r i^{\prime}}\left(t_{1}^{-}\right)-\frac{\eta_{i^{\prime}}}{\Theta_{i^{\prime}}} \operatorname{sign}\left(s_{i^{\prime}}\left(t_{1}^{-}\right)\right)$

$\dot{s}_{i^{\prime} \mid j}\left(t_{1}^{+}\right) / \Theta_{i}^{\prime}=u_{r j}\left(t_{1}^{-}\right)-u_{r i^{\prime}}\left(t_{1}^{-}\right) \leq 0$.

On the other hand, $u_{r j}\left(t_{1}^{+}\right)-u_{r i^{\prime}}\left(t_{1}^{+}\right)>0$, so

$\frac{\eta_{i^{\prime}}}{\Theta_{i}^{\prime}} \operatorname{sign}\left(s_{i^{\prime}}\left(t_{1}^{+}\right)\right)=u_{r j}\left(t_{1}^{+}\right)-u_{r i^{\prime}}\left(t_{1}^{+}\right)-\frac{\dot{s}_{i^{\prime} \mid j}\left(t_{1}^{+}\right)}{\Theta_{i^{\prime}}}>0$.

Since $s_{i^{\prime}}\left(t_{1}\right)=0$, inequality (31) predicts a non-positive sign for $s_{i^{\prime}}\left(t_{1}^{+}\right) / \Theta_{i^{\prime}}$, while inequality (32) indicates a positive sign for the same quantity. This contradiction implies that $s_{j}\left(t_{1}\right)=0$ must hold. This behavior can be clearly observed in any simulation involving the max-min selector.

The following counterpart to Lemma 9 will be instrumental to the stability proof with max-min selection:

Lemma 10. Suppose $t_{1}>0, i \in L$ and $j \in H$ exist which satisfy the conditions of Lemma 9. Suppose $q(t)=j$ for $t<t_{2}$ and $q(t)=i$ for $t \geq t_{2}$, for some $t_{2}>t_{1}$. That is, suppose a mode change exists at $t_{2}$ from a max-selected regulator $j$ to a min-selected regulator $i$ after the opposite change has occurred at $t_{1}$. Then $s_{j}\left(t_{2}\right)=0$.

Proof. The same arguments used in the proof of Lemma 9 apply. In this case, letting $i^{\prime}=\arg \min _{l \in L}\left\{u_{r l}(t)\right\}$ for $t_{1}<t<t_{2}$ and $j^{\prime}=\arg \max _{h \in H}\left\{u_{r h}(t)\right\}$ for $t \geq t_{2}$, one has:

$u_{r i^{\prime}}\left(t_{2}^{-}\right)<u_{r j}\left(t_{2}^{-}\right)$

$u_{r i^{\prime}}\left(t_{2}^{+}\right) \geq u_{r j}\left(t_{2}^{+}\right)$.

The proof of Lemma 9 applies by switching indices $i^{\prime}$ and $j$.

Lemmas 9 and 10 provide key information about the operation of the proposed control system. Of particular importance are the facts that they provide bounds on the number of switchings between the $L$ and $H$ sets. They also imply the existence of a finite time after which all switchings are restricted to the min set, which reduces the proof of stability to the min case.

Proposition 11. Suppose $t_{1}>0, t_{2}>0, i \in L$ and $j \in H$ exist which satisfy the conditions of Lemma 10. Then $q\left(t_{3}\right) \neq j$ for all $t_{3}>t_{2}$.

Proof. After $i$ becomes active, one has

$\dot{s}_{j \mid i}\left(t_{2}^{+}\right) / \Theta_{j}=u_{r i}\left(t_{2}^{+}\right)-u_{r j}\left(t_{2}^{+}\right)-\eta_{j} \operatorname{sign}\left(s_{j}\left(t_{2}^{+}\right)\right) / \Theta_{j}$

with $s_{j}\left(t_{2}^{+}\right)=0$ by Lemma 10 . Since $u_{r i} \geq u_{r j}$ while $i$ is active:

$\dot{s}_{j \mid i}\left(t_{2}^{+}\right) / \Theta_{j} \geq 0$

on the boundary $s_{j}=0$. Suppose $\Theta_{j}>0$. Then $\dot{s}_{j}>0$ as it abandons the boundary $s_{j}=0$. A return to $q=j$ requires $s_{j}=0$, by Lemma 9 . For this to happen, it is necessary that $\dot{s}_{j}<0$ immediately prior to reaching $s_{j}=0$. However, this is not possible due to inequality (33). Thus any return to a regulator belonging to the $H$ set must be such that $q \neq j$. The same conclusion is reached for the case $\Theta_{j}<0$.

Theorem 12. Under Assumptions 1 and 2, all trajectories of System (2), (3) under control input (7) and the max-min switching law converge asymptotically to the unique equilibrium point $\bar{x}_{a i *}$.

Proof. Suppose first that all mode switchings occur only among the $L$ set. Then Theorem 8 proves this theorem. Now suppose that the active regulator belongs to the $H$ set at some time. If all subsequent switchings occur within this set, the system behaves as if it only had the max selector. This implies that an ending regulator other than $i^{*}$ exists, which is a contradiction. Therefore a switch to a regulator in $L$ must occur. According to Proposition 11, a return to the $H$ set may occur only if max regulators still exist that have not been active before. Switchings between the $H$ and $L$ sets may occur until all max regulators have been "used up". Then the $L$ set becomes invariant and switchings are restricted to it, so behavior reduces to that of a min-only system. Theorem 8 then provides the desired result.

Theorem 12 implies that the total number of switchings from the $L$ set to the $H$ set is at most equal to the number of regulators in the $H$ set. 


\section{Invariance properties: limit protection}

The results of this section show that the min, max and max-min designs actually maintain outputs within limits. When the min switching law is used alone, outputs whose $\Theta$ is positive will be protected against upper-limit violations and outputs whose $\Theta$ is negative will be protected against lower-limit violations. Conversely, the max switching law alone protects outputs whose $\Theta$ is positive against lower-limit violations and outputs whose $\Theta$ is negative against upper-limit violations. A max-min scheme is used to cover additional combinations of signs of $\Theta$ and upper or lower limits. An interval $(-\infty, b]$ is invariant for a generic real variable $z(t)$ if $\dot{z}(t) \leq 0$ at $z=b$. Similarly, an interval $[a, \infty)$ is invariant if $\dot{z}(t) \geq 0$ at $z=a$. When an interval is invariant and $z\left(t_{1}\right)$ belongs to the interval for some $t_{1}>0$, then $z(t)$ will remain in the interval for $t \geq t_{1}$. For the proposed technique to be effective, the interval $(-\infty, 0]$ must be invariant for the $s_{j}$ of upper-limited variables, in view of the definition of $s_{j}$ for limited output $y_{j}$. Conversely, $[0, \infty)$ must be invariant for the $s_{j}$ of lower-limited variables.

\subsection{Invariance under min switching}

Let $y_{j}$ be a limited variable. The derivative of $s_{j}$ when $i$ is active is given by Eq. (15). When $i$ is active we must have $u_{r i} \leq u_{r j}$, so:

$\frac{\dot{s}_{j \mid i}}{\Theta_{j}}=\Gamma_{j, i} \tilde{x}_{a}-\frac{\eta_{i}}{\Theta_{i}} \operatorname{sign}\left(s_{i}\right) \leq-\frac{\eta_{j}}{\Theta_{j}} \operatorname{sign}\left(s_{j}\right)$.

Noting that the inequality changes to equality for $j=i$, it is clear that $\frac{\dot{s}_{j}}{\Theta_{j}} \leq 0$ at $s_{j}=0$ under any regulator. If $\Theta_{j}>0$, upperlimit protection is guaranteed. If $\Theta_{j}<0$, lower-limit protection is guaranteed.

\subsection{Invariance under max switching}

Following the same reasoning used for the min case, it is clear that $\frac{\dot{s}_{j}}{\Theta_{j}} \geq 0$ at $s_{j}=0$ under any regulator. If $\Theta_{j}>0$, lowerlimit protection is guaranteed. If $\Theta_{j}<0$, upper-limit protection is guaranteed.

\subsection{Invariance under max-min switching}

One would expect that the max-min arrangement guarantee invariance of any real interval $[a, b]$ containing zero, regardless of the sign of $\Theta$, but this is not the case. An exception occurs for $s_{j}$ when $j \in L$ and the active regulator belongs to $H$. This lack of symmetry arises from the fact that for $q \in H$ to be active it is necessary that $u_{r q}$ be greater than the minimum of all $u_{r l}, l \in L$, but not for every $u_{r l}$ in $L$. By contrast, for $q \in L$ to be active, $u_{r l}$ must be greater than every $u_{r h}, h \in H$. Indeed, suppose $q=i \in L$ is active and consider a variable $s_{j}$ and its derivative along the boundary $s_{j}=0$ :

$\dot{s}_{j \mid i} / \Theta_{j}=u_{r i}-u_{r j}-\eta_{j} \operatorname{sign}\left(s_{j}\right) / \Theta_{j}=u_{r i}-u_{r j}$.

If $j \in L$, it is necessary that $u_{r i}-u_{r j} \leq 0$, while one must have $u_{r i}-u_{r j} \geq 0$ if $j \in H$. Thus, while $q \in L, s_{j}$ is upper-bounded by zero if $\Theta_{j}>0$, and it is lower-bounded by zero if $\Theta_{j}<0$. Now consider $q=i \in H$ to active. Eq. (34) still applies. If $j \in H$ it is necessary that $u_{r i}-u_{r j} \geq 0$. Thus, while $q \in H$, all variables $s_{j}$ associated to the max selector will be upper-bounded by zero when $\Theta_{j}<0$ and will be lower-bounded by zero if $\Theta_{j}>0$. The difficulty arises when considering $j \in L$ while the active regulator is in $H$. The difference $u_{r i}-u_{r j}$ may be positive, negative or zero, and invariance does not apply. Fortunately, separate arguments can be made which maintain the validity of the approach under commonly-found circumstances. These arguments are elaborated in the next section.
Table 1

Guidelines for the association of regulators to selectors.

\begin{tabular}{lll}
\hline Limit & Sign of $\Theta$ & Selector \\
\hline Upper & + & Min \\
Upper & - & Max \\
Lower & + & Max \\
Lower & - & Min \\
Regulated output & N.a. & Min \\
\hline
\end{tabular}

\section{Additional considerations}

For the remainder of the article, it is assumed that regulators are assigned to selectors so as to exploit the invariance properties described above. These assignment rules have been summarized in Table 1.

\subsection{Consistency among limited outputs}

The results of this paper are directly applicable to setpoint changes, implying that initial and final plant states $\left[x^{T} \mid u\right]^{T}$ are equilibrium points. Then it is always possible to re-define variables so that the initial input $u$, state $x$ and outputs $y_{j}$ are zero. Frequently, it occurs that the sign of the DC gain of the transfer functions from $u$ to $y$ for the limited outputs coincides with the sign of $\Theta$. The steady plant input-output relationships have the form

$\bar{y}_{j}=\Theta_{j}\left(1-G_{j} A^{-1} B / \Theta_{j}\right) \bar{u}$

for $j=1 \ldots h$. If $1-G_{j} A^{-1} B / \Theta_{j}>0$, then the sign of steady input $\bar{u}$ will match that of the limit $\bar{y}_{j}$ when $\Theta_{j}>0$ and will be of the opposite sign when $\Theta_{j}<0$. This has useful implications for the behavior of min-variables when $q \in H$, where invariance was not found. The following heuristic reasoning applies: if $q \in H$ because an upper-limited variable from the max group is reaching its (positive) limit, then $u$ will be negative, since $\Theta_{j}$ must be negative according to the assignment rules. Any $y_{i}$ among the minselected variables which is upper-limited will be driven away from its limit by the negative $\bar{u}$, since $\Theta_{i}>0$ by the assignment rules. The same reasoning can be followed for other combinations. This behavior is confirmed in simulation.

\subsection{Outputs with $\Theta=0$}

When a limited output $y_{j}$ is such that $\Theta_{j}=0$, the proposed technique cannot be applied directly, since the sliding functions $s_{j}$ will have relative degree 2 with respect to the control input $u_{r}$. Two ways to overcome this difficulty are available: using second-order sliding modes (Boiko et al., 2007; Boiko, Fridman, \& Castellanos, 2004; Wang, Xu, \& Chen, 2007), and using a nonintegral control law (i.e., eliminating input integration and using $u_{i}$ as control input). The first approach has the advantage of reducing or eliminating the control chattering associated with standard SMC laws, but laws such as the so-called "super-twisting algorithm" (Levant, 2003) have the significant disadvantage of requiring the on-line computation of $\dot{s}_{j}$. The second approach is straightforward to formulate if applied to all regulators. It is also possible to implement integration before the max-min selection for regulators having $\Theta_{i} \neq 0$. Such $u_{i}$, together with the nonintegrated inputs are then applied to the max-min selectors. The results of this paper become applicable after some modifications.

\subsection{Singular $A$}

If $A$ is singular due a single zero eigenvalue, the corresponding integrator can be factored out of the transfer function from $u$ to $y_{j}$ and a new state-space realization can be found where $A$ is nonsingular. The integrator is then shifted to the controller 
to match the assumptions of this paper for design purposes. A practical controller implementation would, of course, omit the explicit integrator, injecting $u_{r}$ directly to the plant.

\subsection{Effect of disturbance}

Suppose a disturbance input is added to Eq. (2):

$\dot{x}=A x+B u+B_{1} \delta$

where $B_{1}$ is an $n$-by- 1 vector and $\delta(t)$ is an unmeasurable exogenous input such that $|\delta(t)| \leq \bar{\delta}$. The input $\delta(t)$ represents an unmatched disturbance if $B_{1}$ does not belong to the column space of $B$ (Edwards \& Spurgeon, 1998; Slotine \& Li, 1990; Utkin, 1992). This means that $\delta(t)$ cannot be regarded as an additive component to the control input $u$. Consequently, it may not be exactly canceled out by $u$ even if it were known or accurately estimated. The presence of disturbance is likely to require modifications to many results presented in this paper, especially regarding the determination of the final regulator and certain statements about the switching sequences. However, certain limit protection properties are retained, even under unmatched disturbance. Suppose $j$ is the active regulator. Then,

$\dot{s}_{j}=\left(G_{j} B_{1}\right) \delta(t)-\eta_{j} \operatorname{sign}\left(s_{j}\right)$.

Since $\delta(t)$ is bounded, choosing $\eta_{j}$ large enough will create an attractive sliding mode at $s_{j}=0$, implying that $y_{j}$ will not cross its limit. When $i \neq j$ is active, however,

$\dot{s}_{j \mid i}=u_{r i}-u_{r j}-\eta_{j} \operatorname{sign}\left(s_{j}\right) / \Theta_{j}+\left(G_{j} B_{1}\right) \delta(t) / \Theta_{j}$.

Even when a definite sign exists for $u_{r j}-u_{r i}$, the sign of the derivative of $s_{j}$ at the boundary $s_{j}=0$ will be driven by the disturbance and no invariance can be inferred. Thus, invariance holds for variables which approach their limits while their own regulator is active.

\subsection{Design example: aircraft engine control}

The thrust developed by a turbofan engine is frequently controlled by a feedback loop where fuel flow rate is the control input and fan speed is the sensed variable. Thrust cannot be sensed in a reliable way; however, it is linked to fan speed through a static function. Hence, setpoints are given in terms of pre-calculated fan speeds. Consider a two-spool turbofan engine model in the $90,000 \mathrm{lb}$. thrust class, linearized at an altitude of $25,000 \mathrm{ft}$. and Mach number 0.62 (Frederick, DeCastro, \& Litt, 2007). Table 2 summarizes the state and output equilibrium parameters and the allowable limits considered for the example. The linearized model has fan speed increment $x_{1} \triangleq \Delta N_{f}$ and core speed increment $x_{2} \triangleq \Delta N_{c}$ in rpm as states and fuel flow increment $u \triangleq \Delta W_{f}$ in pounds per second (pps) as control input. Defining incremental outputs $\Delta y_{j}$, for $j=2,3,4,5$, the model matrices corresponding to Eq. (2) are as follows:

$$
\begin{aligned}
& A=\left[\begin{array}{cc}
-1.7435 & 0.7462 \\
0.5080 & -2.1737
\end{array}\right] \quad B=\left[\begin{array}{l}
287.6845 \\
891.1333
\end{array}\right] \\
& G_{2}=\left[\begin{array}{ll}
0.0071 & 0.0177
\end{array}\right], \quad \Theta_{2}=-18.4743 \\
& G_{3}=\left[\begin{array}{ll}
0.0244 & -0.2665
\end{array}\right], \quad \Theta_{3}=410.4741 \\
& G_{4}=\left[\begin{array}{ll}
-0.0037 & 0.1599
\end{array}\right] \times 10^{-3}, \quad \Theta_{4}=0.0461 \\
& G_{5}=\left[\begin{array}{ll}
0.0017 & 0.0855
\end{array}\right], \quad \Theta_{5}=25.5719
\end{aligned}
$$

where $A$ satisfies Assumption 1. The control objective is to achieve an offset-free setpoint change in fan speed while maintaining limited outputs within bounds at all times. The incremental limits are: $y_{2} \geq \underline{y}_{2}=-15 \%, y_{3} \leq \bar{y}_{3}=400^{\circ} \mathrm{R}, y_{4} \leq \bar{y}_{4}=0.15$ and $y_{5} \geq \underline{y}_{5}=-80$ psi. The design process introduces an additional limited variable $y_{1}=G_{1} x+\Theta_{1} u$ and an associated limit $\bar{y}_{1}$ equal to the desired setpoint for the regulated variable, $y_{0}=x_{1}$. The objective is to ensure $i^{*}=1$ for a range of fan speed setpoints, while achieving good transient performance and preventing limit violations. Design freedoms are $G_{1}, \Theta_{1}$ and the switching gains $\eta_{i}$ appearing in the control laws of Eq. (7). It can be verified that outputs $y_{j}$ for $j=2,3,4,5$ are minimum-phase, satisfying Assumption 2. Five regulators are to be implemented following Eq. (7) and the max-min selection rule of Eq. (17). Regulators are now associated with the min or max selectors according to Table 1. Since $\Theta_{2}<0$ and $y_{2}$ is lower-limited, $u_{r 2}$ is applied to the min selector. Similarly, since $\Theta_{3}$ and $\Theta_{4}$ are positive and $y_{3}$ and $y_{4}$ are upper-limited, the corresponding regulators are applied to the min selector. Also, $\Theta_{5}>0$ and $y_{5}$ is lower-limited, so the regulator is associated with the max selector. Finally, the main regulator is associated with the min selector to match the assumption that $i^{*} \in L$ (Section 4.3). While the choice of $\Theta_{1}$ is made arbitrarily, several methods exist for the selection of sliding coefficients $G_{1}$ (Edwards \& Spurgeon, 1998; Spurgeon, 1992; Utkin, 1992). In this example, $G_{1}$ is designed to match a speed-of-response requirement for the isolated fan speed regulator in the sliding mode. This is achieved by using $G_{1}$ to target specific values or regions for the eigenvalues of $A_{e q, 1}$. In this example, a spectrum of $\{-5.2566$, - 4.3992\} for $A_{e q, 1}$ is obtained with $G_{1}=$ [0.0118 0.0026]. The final step is to ensure that $i^{*}=1$ for the nominal range of setpoint changes. Since the only controller parameters left to be specified are the switching gains $\eta_{i}>0$, and because their choice does not compromise stability, tuning is straightforward and requires little or no iteration. The $\eta$ gains are adjusted until the conditions of Proposition 5 hold for $i^{*}=1$. The selection of the $\eta_{i}$ so that $i^{*}=1$ is not unique. However, some choices result in switching sequences leading to poor transient performance of the regulated variable. The designer must re-adjust $\eta_{i}$ until a satisfactory transient response is observed. For this example, it can be verified that setting $\eta_{1}=20$ and $\eta_{i}\left|\Theta_{i}\right|=15$ for $i=2,3,4,5$ results in $i^{*}=1$. The setpoint for fan speed is first set at $\Delta N_{f}=340$ $\mathrm{rpm}$. If limit regulators are not used, and the main regulator is used in a standard SMC loop, a transient response with a settling time near $1 \mathrm{~s}$, zero overshoot and zero steady-state error is obtained, as seen in Fig. 2 (thin trace), reflecting the nominal performance associated with the choices of $G_{1}$ and $\eta_{1}$. However, simulation shows that three of the limited outputs, namely $\Delta T_{48}, \Delta \mathrm{EPR}$ and $\triangle$ HPC-SM peak beyond their limits in the transient regime. In particular, $\Delta T_{48}$ peaks at $1100^{\circ}$ R. Figs. 2 and 3 show the responses obtained when all limit regulators are used. It can be seen that $T_{48}$ and HPC-SM now "ride" their limits during the transient regime. Naturally, the fan speed response must be slower, but its ability to reach the setpoint will not be hindered, since the design ensures that $i^{*}=1$ under setpoint commands in a range. Note that the transfer function from $\Delta W_{f}$ to $\Delta$ Ps30 has a DC gain of the opposite sign as the transfer function from $\Delta W_{f}$ to $\Delta N_{f}$, causing $\Delta$ Ps30 to move away from its lower-limit. The settling time is now about $1.4 \mathrm{~s}$, showing how this technique conveniently balances control and limit protection tasks. The limit $\Delta T_{48}=400^{\circ} \mathrm{R}$ was made significantly smaller than the incremental peak observed when no limit protection is used. Considering that the absolute limit on $T_{48}$ used in real engine controls is close to $2200^{\circ} \mathrm{R}$ (Litt, Frederick, \& Guo, 2009), $\Delta T_{48}$ could have been chosen as high as $657^{\circ} \mathrm{R}$. Under these conditions, the same design would result in a faster settling time. Fig. 2 also shows the switching history and the sliding variables. The latter have been scaled for graphical convenience, and $s_{5}$ has been omitted since the $\Delta$ Ps30 regulator does not become active. To illustrate activation of the lower limit, suppose now that fan speed is to be reduced, that is, the setpoint is $\Delta N_{f}=-340 \mathrm{rpm}$. It can be verified that $i^{*}=1$ and $i_{0}=1$ still hold under the same design parameters. Figs. 4 and 5 show 
Table 2

Engine equilibrium values at 25,000 ft, Mach 0.62 and $W_{f}=1.67 \mathrm{pps}$.

\begin{tabular}{llll}
\hline Variable & Eq. value & Units & Limit \\
\hline Fan speed, $N_{f}$ & 1915 & $\mathrm{rpm}$ & - \\
Core speed, $N_{c}$ & 8006 & $\mathrm{rpm}$ & - \\
Fuel flow rate, $u=W_{f}$ & 1.67 & pints/s & - \\
High-pressure compressor stall margin, $y_{2}=$ HPC-SM & 29.8 & $\%$ & $\geq 14.8$ \\
High-pressure turbine outlet temperature, $y_{3}=T_{48}$ & 1543 & ${ }^{\circ} \mathrm{R}$ & $\leq 1943$ \\
Engine pressure ratio, $y_{4}=$ EPR & 0.94 & - & $\leq 1.09$ \\
High-pressure compressor outlet pressure, $y_{5}=$ Ps30 & 164.9 & psia & $\geq 84.9$ \\
\hline
\end{tabular}
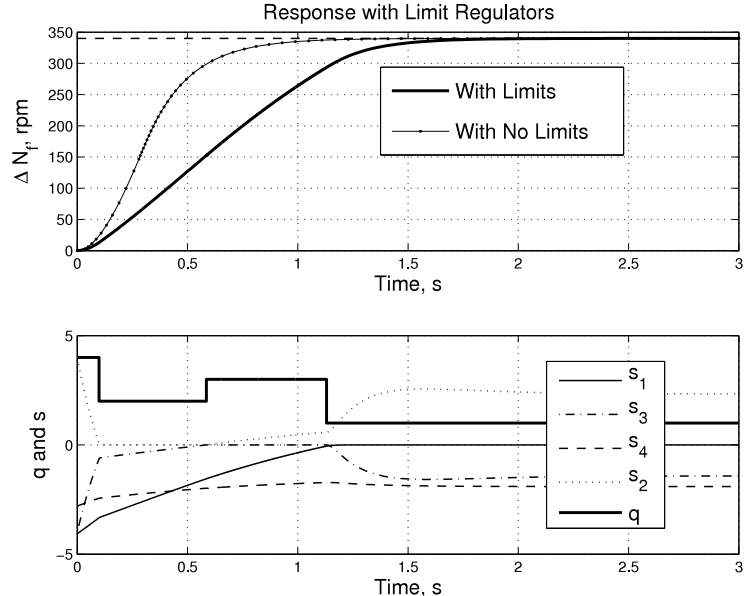

Fig. 2. Fan speed response with limit regulators enabled: positive setpoint change.
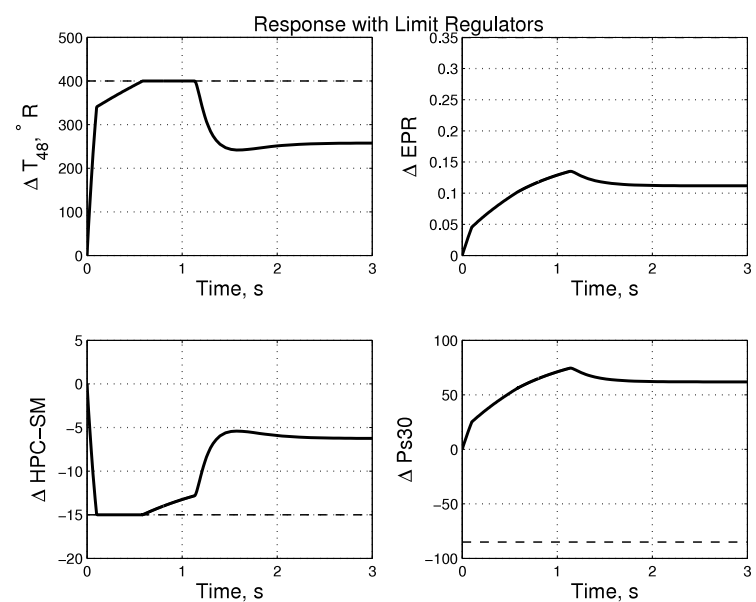

Fig. 3. Limited output response with limit regulators enabled: positive setpoint change.

that $\Delta$ Ps30 reaches its lower limit and holds it for some time. It is observed that $\Delta N_{f}$ responds almost as fast as when the limit regulators are removed. Similar limit protection qualities may be observed upon re-tuning of $\eta_{i}$ or $G_{1}$. The switching history and scaled sliding variables $s_{1}$ and $s_{5}$ are also shown, to illustrate the validity of Lemmas 9, 10 and Proposition 11: only one switching occurs from the $L$ set to the $H$ set, which contains 1 regulator.

\section{Conclusions and final remarks}

A multi-sliding mode regulator scheme with max-min selection logic was proposed. The stability of the min-max arrangement is guaranteed when the individual regulators are designed separately, following the standard sliding mode design procedure. It was established that the min selector protects upper-limited outputs whose direct term is positive and lower-limited outputs
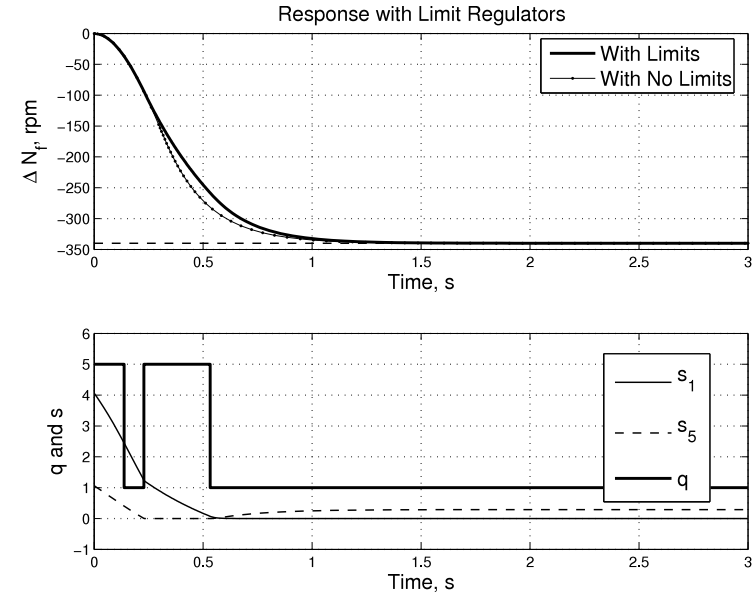

Fig. 4. Fan speed response with limit regulators enabled: negative setpoint change.
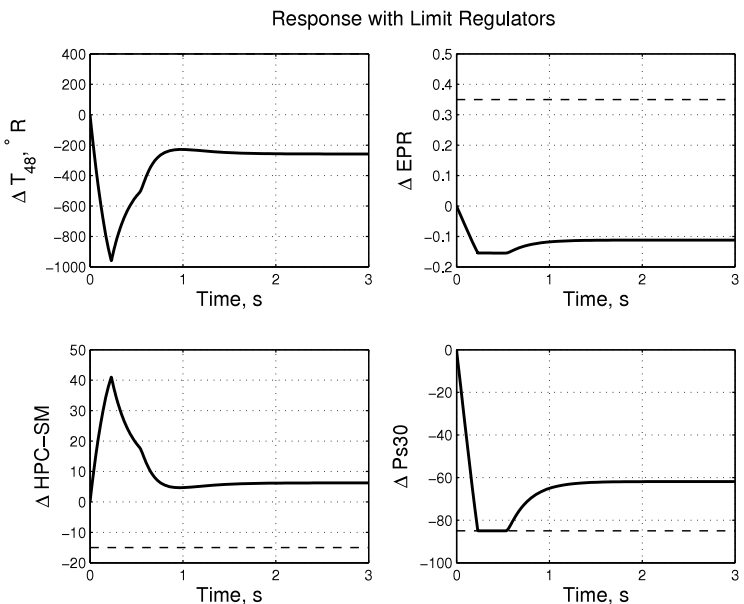

Fig. 5. Limited output response with limit regulators enabled: negative setpoint change.

whose direct term is negative. The max selector offers the opposite protections. When used in combination, the max and min selectors offer additional protection. The case $\Theta=0$ can be accommodated by eliminating input integration or by introducing high-order sliding mode techniques. Invariance in the presence of unmatched disturbance is guaranteed for outputs approaching their limits while their own regulator is active. Some simulations have indicated that disturbance may actually trigger a switch to the regulator whose limited output reaches its limit, motivating further research. Other extensions include the development of multi-input and adaptive versions of the technique.

\section{Acknowledgments}

The author wishes to acknowledge Jonathan Litt and Sanjay Garg from the NASA GRC Controls and Dynamics Branch for 
supporting this research through the NASA Summer Faculty Fellowship Program.

\section{References}

Åstrom, K. J., \& Hägglund, T. (1995). PID controllers: theory, design and tuning. Instrument Society of America.

Bemporad, A., Borrelli, F., \& Morari, M. (2002a). Model predictive control based on linear programming - the explicit solution. IEEE Transactions on Automatic Control, 47(12), 1974-1985.

Bemporad, A., et al. (2002b). The explicit quadratic regulator for constrained systems. Automatica, 38(1), 3-20.

Blanchini, F. (1999). Set invariance in control. Automatica, 35, 1747-1767.

Boiko, I., et al. (2007). Analysis in chattering in systems with second-order sliding modes. IEEE Transactions on Automatic Control, 52(11), 2085-2102.

Boiko, I., Fridman, L., \& Castellanos, M. I. (2004). Analysis of second-order sliding mode algorithms in the frequency domain. IEEE Transactions on Automatic Control, 49(6), 946-950.

Branicky, M. S. (1994). Analysis of continuous switching systems: theory and examples. In Proc. American control conference (pp. 3110-3114).

Branicky, M. S. (1998). Multiple Lyapunov functions and other analysis tools for switched and hybrid systems. IEEE Transactions on Automatic Control, 43(4), 475-482.

Edwards, C., \& Spurgeon, S. (1998). Sliding mode control: theory and applications. Taylor and Francis.

Foss, A. M. (1981). A criterion to assess stability of a lowest-wins control strategy. IEE Proceedings, Part D, 128(1), 1-8

Frederick, D. K., DeCastro, J. A., \& Litt, J. S. (2007). User's guide for the commercial modular aero-propulsion system simulation (CMAPSS). Technical report, NASA TM-2007-215026, Cleveland, Ohio. Glenn Research Center.

Glattfelder, A. H., \& Schaufelberger, W. (2003). Control systems with input and output constraints. In Advanced textbooks in control and signal processing. Springer.

Jaw, L. (2009). Aircraft engine controls: design, system analysis, and health monitoring. In AIAA Education Series.

Johansson, M. (2003). Piecewise linear control systems. In Lecture notes in control and information sciences: Vol. 284. Springer.

Levant, A. (2003). Higher-order sliding modes, differentiation and output feedback control. International Journal of Control, 76, 924-941.
Litt, J. S. et al. (2009). The case for intelligent propulsion control for fast engine response. In AIAA infotech@aerospace conference, AIAA paper AIAA-20091876.

Litt, J. S., Frederick, D. K., \& Guo, T. -H. (2009). The case for intelligent propulsion control for fast engine response. In Proc. AIAA infotech aerospace conference.

Richter, H. (2010). Motion control of a container with slosh: constrained sliding mode approach. ASME Journal of Dynamic Systems, Measurement and Control, 132(3).

Richter, H., O’Dell, B. D., \& Misawa, E. A. (2007). Robust positively invariant cylinders in constrained variable structure control. IEEE Transactions on Automatic Control, 52(11), 2058-2069.

Richter, H., Singaraju, A. V., \& Litt, J. S. (2008). Multiplexed predictive control of a large commercial turbofan engine. AIAA Journal of Guidance, Dynamics and Control, 31(2), 273-281.

Slotine, J., \& Li, W. (1990). Applied nonlinear control. Prentice Hall.

Spang, H. A., \& Brown, H. (1999). Control of jet engines. Control Engineering Practice, 7, 1043-1059.

Spurgeon, S. K. (1992). Hyperplane design techniques for discrete-time variable structure control. International Journal of Control, 55, 445-456.

Utkin, V. I. (1992). Sliding modes in control optimization. Springer-Verlag.

Wang, N., Xu, W., \& Chen, F. (2007). Output feedback second-order sliding-mode control of uncertain linear systems with relative degree 2. IET Control Theory and Applications, 1(4), 880-886.

Zhao, J., \& Hill, D. J. (2008). On stability, L2-gain and $\mathscr{H}_{\infty}$ control for switched systems. Automatica, 44(5), 1220-1232. 